\title{
MINIREVIEW
}

\section{The genetics of generalized vitiligo: autoimmune pathways and an inverse relationship with malignant melanoma}

\author{
Richard A Spritz*
}

\begin{abstract}
Generalized vitiligo (GV) is the most common pigmentation disease, in which white spots of skin and overlying hair result from loss of melanocytes from the involved regions. GV is a complex disease involving both genetic predisposition and unknown environmental triggers. Whereas various pathogenetic mechanisms have been suggested, most evidence supports an autoimmune basis for this disease. Recently, three different genome-wide association studies of GV have been reported, identifying a total of 17 confirmed GV susceptibility loci. Almost all of these genes encode immunoregulatory proteins, together highlighting pathways by which melanocytes might be recognized and killed. Moreover, the biological interaction between two of these GV susceptibility genes, HLA-A and TYR (encoding tyrosinase), points to an apparent inverse relationship between susceptibility to $G V$ versus malignant melanoma, suggesting that GV may result, in part, from dysregulation of normal processes of immune surveillance against melanoma.
\end{abstract}

\section{The genetics and epidemiology of generalized vitiligo}

Generalized vitiligo (GV) is the most common depigmentation disorder, in which acquired multifocal patches of white skin and overlying hair result from loss of melanocytes in the involved areas (Figure 1) [1,2]. The prevalence of $\mathrm{GV}$ is approximately $0.5 \%$ in various populations [3], with an average age of onset at about 24 years and occurring with approximately equal frequency in males and females [4]. The concordance of GV in monozygotic twin-pairs is approximately $23 \%$ [4], and

*Correspondence: richard.spritz@ucdenver.edu

Human Medical Genetics Program, University of Colorado School of Medicine, Aurora, CO 80045, USA epidemiological evidence indicates that GV is a complex trait involving multiple genes and unknown environmental factors [3].

Many hypotheses have been proposed to account for melanocyte loss in GV [1]. Most current evidence supports an autoimmune basis of disease [5], though the triggers of the autoimmune response remain unknown. Among European-derived white individuals with GV, about $15 \%$ to $25 \%$ have at least one additional concomitant autoimmune disease, particularly autoimmune thyroid disease (Hashimoto's thyroiditis and Graves' disease), pernicious anemia, rheumatoid arthritis, psoriasis, type 1 diabetes, Addison's disease and systemic lupus erythematosus; these diseases also occur at increased frequencies in first-degree relatives of patients with GV, whether or not those relatives have GV themselves [4]. Together, these findings indicate that patients with GV and their close relatives have inherited susceptibility to this specific diathesis of autoimmune diseases, mediated by shared susceptibility genes.

\section{Vitiligo susceptibility genes}

Candidate gene association studies and gene expression analyses have produced a long list of GV candidate genes, of which only HLA (human leukocyte antigen) and PTPN22 (protein tyrosine phosphatase, non-receptor type 22) have had consistent support from multiple studies; most of the rest are likely to represent false positives $[3,6]$. Two additional genes, NALP1 (now NLRP1; NLR family, pyrin domain containing 1) and XBP1 (X-box binding protein 1 ), were first mapped by unbiased genome-wide linkage analyses and subsequently were identified by positionally targeted genetic association studies $[7,8]$; both of these genes have subsequently been replicated by multiple studies $[3,6]$. These four confirmed GV susceptibility loci - HLA, PTPN22, NLRP1 and XBP1 all encode important immunoregulatory proteins, lending support to the autoimmune hypothesis of GV pathogenesis.

Recently, three different genome-wide association studies (GWASs) of GV have been reported: two from 


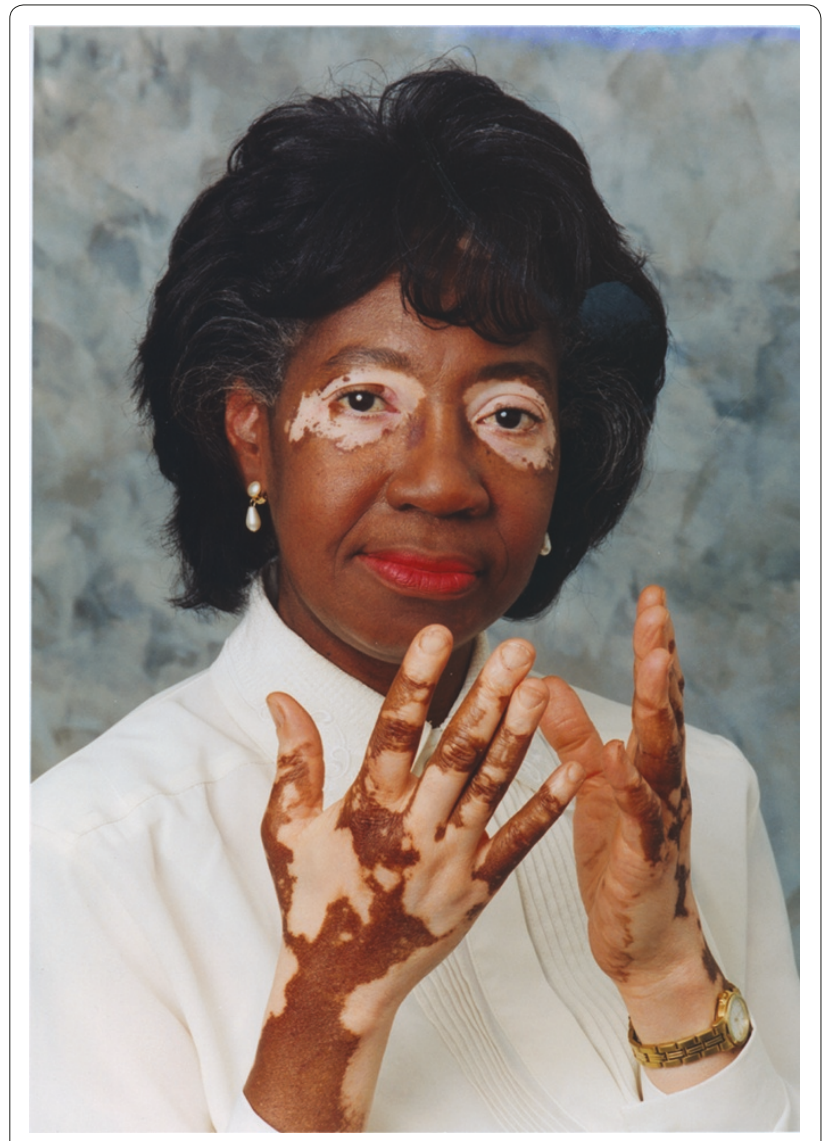

Figure 1. A patient with generalized vitiligo. Note the typical pattern of bilateral, multifocal depigmentation of hands and periorbital regions.

European-derived white populations [9-11] and one from China [12]. Together, these studies identified a total of 17 confirmed GV susceptibility loci, yielding major insights into pathways of disease pathogenesis and overall strongly supporting an autoimmune basis for typical GV. The first GWAS, of a founder population in an isolated Romanian village with a high prevalence of GV and other autoimmune diseases [9], detected association at chromosome 6qter near IDDM8, which is a type 1 diabetesrheumatoid arthritis locus in the vicinity of $S M O C 2$ (SPARC-related modular calcium binding 2). The second GWAS [10,11], also carried out in European-derived white individuals, identified a total of 13 susceptibility loci for $\mathrm{GV}$, including $H L A$ class I (specifically, HLA-A*0201), HLA class II, PTPN22, RERE (arginineglutamic acid dipeptide repeats), FOXP1 (forkhead box P1), LPP (LIM domain containing preferred translocation partner in lipoma), CCR6 (chemokine (C-C motif) receptor 6), IL2RA (interleukin 2 receptor, $\alpha$ ), TYR, GZMB (granzyme B), NLRP1, UBASH3A (ubiquitin-associated and $\mathrm{SH} 3$ domain containing $\mathrm{A}$ ) and C1QTNF6 (C1q and tumor necrosis factor related protein 6). Moreover, subsequent re-analysis of this genome-wide dataset to specifically test association of 33 biological candidate genes previously implicated in GV identified three additional GV susceptibility loci: TSLP (thymic stromal lymphopoietin), XBP1 and FOXP3 (forkhead box P3) [6]. The third GWA study [12], carried out in a Chinese population, also identified GV susceptibility loci in the $H L A$ class I and class III regions, and likewise detected association with CCR6. Moreover, the GV-associated SNP in the CCR6 region is the same in European-derived white individuals and Chinese people, suggesting that these two populations may share a single causal allele, and is located only $1.44 \mathrm{Mb}$ from the previous GVassociated SNP in the SMOC2 region [9], suggesting the possibility that these two signals might be related. Virtually all of the confirmed GV susceptibility loci encode known immunoregulatory proteins, and many have been associated with genetic susceptibility to other autoimmune diseases that are epidemiologically linked to GV (Figure 2).

The one exception among the susceptibility loci associated with GV is TYR, which encodes tyrosinase, the key enzyme of melanin biosynthesis in melanocytes. However, in GV even $T Y R$ may act primarily to modulate recognition of the melanocyte target cell by the immune system. Beyond its role in pigmentation, tyrosinase is the major autoantigen in GV. In this regard, GV is thus analogous to type 1 diabetes and autoimmune thyroid disease, in that genetic susceptibility to disease involves genes that encode key specialized intracellular components of the autoimmune target cell types and that constitute major autoantigens for the corresponding disease (GV: TYR, tyrosinase; type 1 diabetes: INS, insulin; autoimmune thyroid disease: $T G$, thyroglobulin). For GV, the causal TYR susceptibility variant appears to be the major (Arg) allele of rs1126809, a common non-synonymous (Arg402Gln) polymorphism that has a minor allele frequency of 0.22 to 0.40 in European-derived white individuals. This polymorphism is rare in other populations, which is why it was not detected in the Chinese GWAS, even though TYR may well play a role in GV pathogenesis in all populations. In contrast, the minor (Gln) allele, which is protective with respect to GV, is associated with susceptibility to malignant melanoma in European-derived white individuals $[13,14]$. Thus, from the standpoint of genetic susceptibility, the TYR Arg402Gln polymorphism represents an inverse relationship between GV and malignant melanoma.

Much of the biology that is likely to underlie this inverse relationship is already known, largely from extensive studies on melanoma patients, in whom GV may develop during the course of treatment and is an auspicious prognostic sign. Tyrosinase is a major antigen 


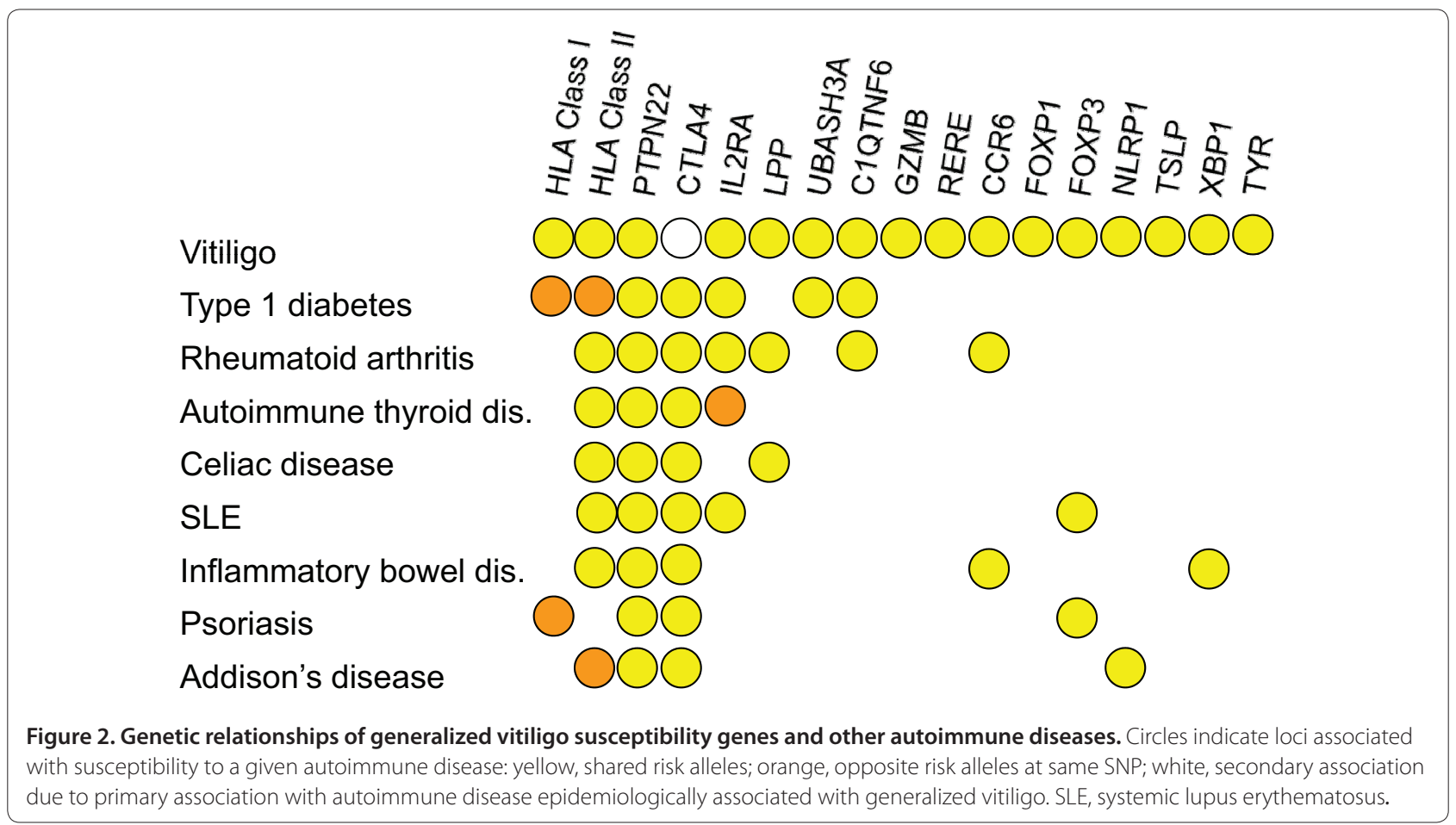

presented to the immune system on the surface of melanocytes and melanoma cells by HLA class I molecules, principally $H L A-A^{*} 0201$, which itself is a major GV risk allele. Indeed, $H L A-A^{*} 0201$ and TYR 402Arg exhibit significant genetic interaction in promoting GV susceptibility [10], reflecting a corresponding biological interaction. One of the important class-restricted epitopes presented by $H L A-A^{*} 0201$ is a specific modified tyrosinase peptide: YMDGTMSQV [15]. However, the TYR 402Gln variant results in an unstable polypeptide that is retained in the endoplasmic reticulum and degraded, thereby reducing the amount of tyrosinase peptide available for presentation on the cell surface. Moreover, presentation of this tyrosinase peptide by $H L A-A^{*} 0201$ requires the posttranslational modification of residue 371Asn to Asp [15], via a mechanism that is probably inefficient in the TYR 402Gln polypeptide. Tyrosinase is thus an important signal by which the immune system recognizes melanocytes, and tyrosinase-402Arg is likely to make a greater contribution than tyrosinase-402Gly to immune surveillance (and thus protection) against malignant melanoma and to susceptibility to GV, whereas tyrosinase-402Gln is associated with lower susceptibility to GV but greater risk of melanoma. Indeed, the odds ratio for vitiligo susceptibility is 2.5 in TYR 402Arg homozygotes compared to 402Gln homozygotes (10).

Interestingly, two of the other GV susceptibility loci, $I L 2 R A$ and $G Z M B$, encode proteins involved in differentiation and effector functions of cytotoxic $\mathrm{T}$ lymphocytes
(CTLs) that mediate melanocyte killing in GV and perhaps also participate in immune surveillance for melanoma cells. Thus, at least four of the GV genome-wide association signals - HLA class I (HLA-A*0201), TYR $402 \mathrm{Arg}, I L 2 R A$ and $G Z M B$ - may be part of a pathway by which CTLs recognize and ultimately kill melanocytes in patients with GV, and perhaps also protect against incipient melanomas.

These observations open several doors to future studies. In GV, it has been known for some time that patients have circulating skin-homing melanocyte-specific CTLs [16]. However, the molecular epitopes recognized by these melanocyte-specific CTLs have not yet been identified. At least in GV patients carrying $H L A-A^{*} 0201$, one of these CTL epitopes might be the modified tyrosinase peptide YMDGTMSQV. Furthermore, it is not yet known whether susceptibility to GV is generic with respect to $H L A-A * 0201$ (which is quite common), or whether it is particular to specific subtypes, and whether those subtypes conversely confer the greatest protection against melanoma.

\section{Conclusions and perspectives}

Recent GWASs have yielded substantial progress in identifying genes involved in risk of GV, with 17 loci now confirmed (HLA class I, HLA class II, HLA class III, PTPN22, RERE, FOXP1, LPP, TSLP, CCR6, IL2RA, TYR, GZMB, NLRP1, UBASH3A, XBP1, C1QTNF6, and FOXP3): 16 in European-derived white individuals and 
four in Chinese people, and, for a few genes, in both. Nevertheless, the 16 loci identified in European-derived white individuals together account for only $10 \%$ of the total genetic risk of GV in that group, indicating that additional loci probably remain to be discovered, with a few common and perhaps numerous rare variants accounting for disease risk at each locus. Essentially all of the confirmed GV susceptibility genes regulate function of the immune system, and many have also been associated with other autoimmune diseases, highlighting shared pathways of autoimmune susceptibility among these diseases.

Furthermore, findings for both HLA-A and TYR suggest an inverse relationship between susceptibility to GV and susceptibility to malignant melanoma, with genetic interaction that reflects underlying biochemical and functional interaction between the corresponding proteins. The overall picture indicates that genetic variation at HLA-A*0201 and TYR interacts to modulate immune surveillance against malignant melanoma, with heightened surveillance predisposing to GV and protecting against melanoma, and reduced surveillance protecting against GV but predisposing to melanoma. This biological relationship may also explain the frequent occurrence of GV in patients treated for melanoma, in whom development of this autoimmune phenotype constitutes a relatively favorable prognostic sign.

While these discoveries underscore the autoimmune nature of GV, they do not offer specific clues as to the environmental triggers that may initiate the autoimmune response. GV is a disease of the skin, the organ that is the first point of contact for one's interaction with the external environment, and which is highly accessible for analysis of that interaction. Furthermore, because GV is so visually evident, it is usually diagnosed early. Thus, identification of GV susceptibility genes may enable identification of individuals at high genetic risk, enabling relatively direct analysis of potentially causal geneenvironment interactions, both retrospectively in patients with relatively recent disease onset, and prospectively in individuals who are at high genetic risk.

\section{Abbreviations \\ CTL, cytotoxic T lymphocyte; GV, generalized vitiligo; GWAS, genome-wide association study; SNP, single-nucleotide polymorphism.}

\section{Competing interests}

The author declares that he has no competing interests.

\section{Author's information}

Dr Richard A Spritz is Professor of Pediatrics and Director of the Human Medical Genetics Program at the University of Colorado School of Medicine. The work of Dr Spritz in the field of human genetics began in the 'premolecular'era, and in the mid-1970s he took part in the first cloning of human genes and identification of the first human disease gene mutation. Over the past 30 years, Dr Spritz and his colleagues have studied the genes involved in causing many different human diseases, including hemoglobin disorders, albinism and other skin diseases, autoimmune diseases such as vitiligo and thyroid disease, and cleft lip/palate, and he has published over 200 scientific papers on these investigations. Most recently, Dr Spritz led an international team that carried out the first genome-wide association study for generalized vitiligo.

\section{Acknowledgements}

This work was supported in part by grants R01 AR45584 and R01 AR056292 from the National Institutes of Health.

Published: 19 October 2010

\section{References}

1. Picardo M, Taïeb A: Vitiligo. Heidelberg: Springer; 2010.

2. Birlea SA, Spritz RA, Norris DA: Vitiligo. In Fitzpatrick's Dermatology in General Medicine. 8th edition. Edited by Wolff K. New York: McGraw-Hill; in press.

3. Spritz RA: Shared genetic relationships underlying generalized vitiligo and autoimmune thyroid disease. Thyroid 2010, 20:745-754.

4. Alkhateeb A, Fain PR, Thody A, Bennett DC, Spritz RA: Epidemiology of vitiligo and associated autoimmune diseases in Caucasian probands and their relatives. Pigment Cell Res 2003, 16:208-214.

5. Le Poole IC, Luiten RM: Autoimmune etiology of generalized vitiligo. Curr Dir Autoimmun 2008, 10:227-243.

6. Birlea SA, Jin Y, Bennett DC, Wallace MR, Herbstman DM, McCormack WT, Kemp EH, Gawkrodger DJ, Weetman AP, Picardo M, Leone G, Taïeb A, Jouary T, Ezzedine K, van Geel N, Lambert J, Overbeck A, Fain PR, Spritz RA: Comprehensive analysis of candidate genes for generalized vitiligo supports XBP1, FOXP3, and TSLP. J Investig Dermatol, in press.

7. Jin Y, Mailloux CM, Gowan K, Riccardi SL, LaBerge G, Bennett DC, Fain PR, Spritz RA: NALP1 in vitiligo-associated multiple autoimmune disease. NEngl J Med 2007, 356:1216-1225.

8. Ren Y, Yang S, Xu S, Gao M, Huang W, Gao T, Fang Q, Quan C, Zhang C, Sun L, Liang Y, Han J, Wang Z, Zhang F, Zhou Y, Liu J, Zhang X: Genetic variation of promoter sequence modulates XBP1 expression and genetic risk for vitiligo. PLoS Genet 2009, 5:e1000523.

9. Birlea SA, Gowan K, Fain PR, Spritz RA: Genome-wide association study of generalized vitiligo in an isolated European founder population identifies SMOC2, in close proximity to IDDM8. J Investig Dermatol 2010, 130:798-803.

10. Jin Y, Birlea SA, Fain PR, Gowan K, Riccardi SL, Holland PJ, Mailloux CM, Sufit AJ, Hutton SM, Amadi-Myers A, Bennett DC, Wallace MR, McCormack WT, Kemp EH, Gawkrodger DJ, Weetman AP, Picardo M, Leone G, Taïeb A, Jouary T, Ezzedine K, van Geel N, Lambert J, Overbeck A, Spritz RA: Variant of TYR and autoimmunity susceptibility loci in generalized vitiligo. New Engl J Med 2010, 362:1686-1697.

11. Jin Y, Birlea SA, Fain PR, Mailloux CM, Riccardi SL, Gowan K, Holland PJ, Bennett DC, Wallace MR, McCormack WT, Kemp EH, Gawkrodger DJ, Weetman AP, Picardo M, Leone G, Taïeb A, Jouary T, Ezzedine K, van Geel N, Lambert J, Overbeck A, Spritz RA: Common variants in FOXP1 are associated with generalized vitiligo. Nat Genet 2010, 42:576-578.

12. Quan $C$, Ren $Y Q$, Xiang LH, Sun LD, Xu AE, Gao XH, Chen HD, Pu XM, Wu RN, Liang CZ, Li JB, Gao TW, Zhang JZ, Wang XL, Wang J, Yang RY, Liang L, Yu JB, Zuo XB, Zhang SQ, Zhang SM, Chen G, Zheng XD, Li P, Zhu J, Li YW, Wei XD, Hong WS, Ye Y, Zhang Y, et al:: Genome-wide association study for vitiligo identifies susceptibility loci at 6q27 and the MHC. Nat Genet 2010, 42:614-618.

13. Gudbjartsson DF, Sulem P, Stacey SN, Goldstein AM, Rafnar T, Sigurgeirsson B, Benediktsdottir KR, Thorisdottir K, Ragnarsson R, Sveinsdottir SG, Magnusson V, Lindblom A, Kostulas K, Botella-Estrada R, Soriano V, Juberías P, Grasa M, Saez B, Andres R, Scherer D, Rudnai P, Gurzau E, Koppova K, Kiemeney LA, Jakobsdottir M, Steinberg S, Helgason A, Gretarsdottir S, Tucker MA, Mayordomo Jl, et al:: ASIP and TYR pigmentation variants associate with cutaneous melanoma and basal cell carcinoma. Nat Genet 2008, 40:886-891.

14. Bishop DT, Demenais F, Iles MM, Harland M, Taylor JC, Corda E, RandersonMoor J, Aitken JF, Avril MF, Azizi E, Bakker B, Bianchi-Scarrà G, Bressac-de Paillerets B, Calista D, Cannon-Albright LA, Chin-A-Woeng T, DebniakT, Galore-Haskel G, Ghiorzo P, Gut I, Hansson J, Hocevar M, Höiom V, Hopper JL, Ingvar C, Kanetsky PA, Kefford RF, Landi MT, Lang J, Lubiński J, et al:: Genomewide association study identifies three loci associated with melanoma risk. Nat Genet 2009, 41:920-925.

15. Skipper JCA, Hendrickson RC, Gulden PH, Britchard V, Van Pel A, Chen Y, Shabanowitz J, Wolfel T, Slingluff, CL Jr, Boon T, Hunt DF, Engelhard VH: An HLA-A2-restricted tyrosinase antigen on melanoma cells results from 
posttranslational modification and suggests a novel pathway for processing of membrane proteins. J Exp Med 1996, 183:527-534.

16. Ogg GS, Dunbar PR, Romero P, Chin J-L, Cerundolo V: High frequency of skin-homing melanocyte-specific cytotoxic T lymphocytes in autoimmune vitiligo. J Exp Med 1998, 188:1203-1208. doi:10.1186/gm199

Cite this article as: Spritz RA: The genetics of generalized vitiligo: autoimmune pathways and an inverse relationship with malignant melanoma. Genome Medicine 2010, 2:78. 